\title{
Azimuthal Angular Distributions in EDDE as spin-parity analyser and glueball filter for LHC.
}

\author{
Petrov V.A., Ryutin R.A., Sobol A.E. \\ Institute for High Energy Physics \\ 142 281 Protvino, Russia \\ and \\ J.-P. Guillaud \\ LAPP, Annecy, France
}

\begin{abstract}
Exclusive Double Diffractive Events (EDDE) are analysed as the source of information about the central system. Experimental possibilities for exotic particles searches are considered. From the reggeized tensor current picture some azimuthal angle dependences were obtained to fit the data from WA102 experiment and to make predictions for LHC collider.
\end{abstract}

\section{Keywords}

Exclusive Double Diffractive Events - Pomeron - Regge-Eikonal model - glueball Higgs 


\section{Introduction}

Since the early time the process of exclusive production of central systems of particles with quasi-diffractively scattered initial particles was considered as an important source of information about high-energy dynamics of strong interactions both in theory and experiment.

If one takes only one particle produced, this is the first "genuinely" inelastic process which not only retains a lot of features of elastic scattering but also shows clearly how the initial energy is being transformed into the secondary particles. General properties of such amplitudes were considered in Ref. [1].

Theoretical consideration of these processes on the basis of Regge theory goes back to papers [2]. Some new interest was related to possibly good signals of centrally produced Higgs bosons and heavy quarkonia [3].

As Pomerons are the driving force of the processes in question it is naturally to expect that glueball production will be favorable, if one believes that Pomerons are mostly gluonic objects [4]. Central glueball production was suggested as possible origin of the total cross section rise in Ref. [5]. One of the early proposal for experimental investigations of centrally produced glueballs in EDDE was made in [6].

As to the most recent experimental studies one has to mention the series of results from the experiment WA102 [7]-[9].

It was proposed in Ref. [10] that the process of single-particle diffractive production can serve as a filter to separate $q \bar{q}$-states from glueballs due to special dependence on azimuthal angle.

In this paper we study the process of single particle production in double diffractive events in the framework of Regge picture (based on Lorentz tensor reggeized exchanges) with account of absorbtion effects both in initial and final state. With parameters fixed from the fit of the WA102 data we give predictions for production of various $J^{P C}$ states at LHC. The main conclusion from WA102 that $q \bar{q}$-states and glueballs have distinct $\phi$-dependence (with account of possible mixing) remains true, in our approach, for LHC energy, though the functional form of $\phi$-distributions changes due to significant absorbtion effects.

\section{EDDE kinematics and cross-sections.}

Here we consider the process $p+p \rightarrow p+X+p$, where $\mathrm{X}$ is a particle or a system of particles, spin and parity of which are fixed. In the kinematics which corresponds to the double Regge limit (see Fig.1) the light-cone representation $(+,-; \perp)$ for momenta of 
colliding and scattered particles is the following:

$$
\begin{aligned}
& p_{1}=\left(\sqrt{\frac{s}{2}}, \frac{m^{2}}{\sqrt{2 s}}, \mathbf{0}\right) \\
& p_{2}=\left(\frac{m^{2}}{\sqrt{2 s}}, \sqrt{\frac{s}{2}}, \mathbf{0}\right) \\
& p_{1}^{\prime}=\left(\left(1-\xi_{1}\right) \sqrt{\frac{s}{2}}, \frac{\boldsymbol{\Delta}_{1}^{2}+m^{2}}{\left(1-\xi_{1}\right) \sqrt{2 s}},-\boldsymbol{\Delta}_{1}\right) \\
& p_{2}^{\prime}=\left(\frac{\boldsymbol{\Delta}_{2}^{2}+m^{2}}{\left(1-\xi_{2}\right) \sqrt{2 s}},\left(1-\xi_{2}\right) \sqrt{\frac{s}{2}},-\boldsymbol{\Delta}_{2}\right) .
\end{aligned}
$$

$\xi_{1,2}$ are fractions of protons' momenta carried by reggeons. The boldface type is used for two-dimensional transverse vectors. From the above notations we can obtain the relations:

$$
\begin{aligned}
t_{1,2} & =\Delta_{1,2}^{2} \simeq-\frac{\Delta_{1,2}^{2}\left(1+\xi_{1,2}\right)+\xi_{1,2}^{2} m^{2}}{1-\xi_{1,2}} \simeq-\Delta_{1,2}^{2}, \xi_{1,2} \rightarrow 0 \\
\cos \phi_{0} & =\frac{\boldsymbol{\Delta}_{1} \boldsymbol{\Delta}_{2}}{\left|\boldsymbol{\Delta}_{1}\right|\left|\boldsymbol{\Delta}_{2}\right|} \\
M_{\perp}^{2} & =\xi_{1} \xi_{2} s \simeq M_{X}^{2}+\left|t_{1}\right|+\left|t_{2}\right|+2 \sqrt{t_{1} t_{2}} \cos \phi_{0}, 0 \leq \phi_{0} \leq \pi \\
\left(p_{1}\right. & \left.+\Delta_{2}\right)^{2}=s_{1} \simeq \xi_{2} s \\
\left(p_{2}\right. & \left.+\Delta_{1}\right)^{2}=s_{2} \simeq \xi_{1} s \\
s_{1} s_{2} & =s M_{\perp}^{2}
\end{aligned}
$$

Physical region of double diffractive events is defined by the following kinematical cuts:

$$
\begin{gathered}
0.01 \mathrm{GeV}^{2} \leq\left|t_{1,2}\right| \leq 1 \mathrm{GeV}^{2}, \\
\xi_{\min } \simeq \frac{M_{X}^{2}}{s \xi_{\max }} \leq \xi_{1,2} \leq \xi_{\max }=0.1 .
\end{gathered}
$$

The discussion on the choice of cuts (3)-(4) for diffractive events and references to other authors were given in [11],[12]. We can write the relations in terms of $y_{1,2}$ and $y_{X}$ (rapidities of hadrons and the system X correspondingly). For instance:

$$
\begin{aligned}
\xi_{1,2} & \simeq \frac{M_{X}}{\sqrt{s}} e^{ \pm y_{X}} \\
\left|y_{X}\right| & \leq y_{0}=\ln \left(\frac{\sqrt{s} \xi_{\text {max }}}{M_{X}}\right) \\
\left|y_{1,2}\right| & =\frac{1}{2} \ln \frac{\left(1-\xi_{1,2}\right)^{2} s}{m^{2}-t_{1,2}} \geq 9
\end{aligned}
$$

The cross-section of this process can be written as

$$
\frac{d \sigma}{d t_{1} d t_{2} d \phi_{0} d y_{X}} \simeq \frac{\pi\left|T_{p p \rightarrow p X p}^{\text {Unit. }}\right|^{2}}{8 s^{2}(2 \pi)^{5}},
$$


where $T_{p p \rightarrow p X p}^{\text {Unit. }}$ is the amplitude of the process, which can be calculated from the "bare" amplitude of Fig.1 by the unitarization procedure depicted in Fig.2, where

$$
\begin{aligned}
& T_{X}=T_{p p \rightarrow p X p}, \\
& V\left(s, \mathbf{q}_{T}\right)=4 s(2 \pi)^{2} \delta^{2}\left(\mathbf{q}_{T}\right)+4 s \int d^{2} \mathbf{b} e^{i \mathbf{q}_{T} \mathbf{b}}\left[e^{i \delta_{p p \rightarrow p p}}-1\right] \text {, } \\
& T_{X}^{U n i t .}\left(p_{1}, p_{2}, \Delta_{1}, \Delta_{2}\right)=\frac{1}{16 s s^{\prime}} \int \frac{d^{2} \mathbf{q}_{T}}{(2 \pi)^{2}} \frac{d^{2} \mathbf{q}_{T}^{\prime}}{(2 \pi)^{2}} V\left(s, \mathbf{q}_{T}\right) \text {. } \\
& \text { - } T_{X}\left(p_{1}-q_{T}, p_{2}+q_{T}, \Delta_{1 T}, \Delta_{2 T}\right) \cdot V\left(s^{\prime}, \mathbf{q}_{T}^{\prime}\right) \text {, } \\
& \Delta_{1 T}=\Delta_{1}-q_{T}-q_{T}^{\prime}, \\
& \Delta_{2 T}=\Delta_{2}+q_{T}+q_{T}^{\prime},
\end{aligned}
$$

and $\delta_{p p \rightarrow p p}$ can be found in Ref. [13]. Left and right parts $V$ represent "soft" rescattering effects for initial and final states, i.e. multi-Pomeron exchanges. These "outer" unitarity corrections can reduce the integrated cross-section by the factor, which is determined by kinematical cuts and the nature of the produced system $\mathrm{X}$.

\section{Calculation of the "bare" amplitude.}

In order to calculate peculiar momentum transfers and azimuthal angle dependence we use the amplitudes with meson exchanges of arbitrary spins with subsequent reggeization.

Basic elements of such approach are vertex functions

$$
T^{\mu_{1} \ldots \mu_{J}}(p, \Delta)=<p-\Delta\left|I^{\mu_{1} \ldots \mu_{J}}\right| p>
$$

and

$$
\begin{aligned}
& F_{\alpha_{1} \ldots \alpha_{J}}^{\mu_{1} \ldots \mu_{J_{1}}, \nu_{1} \ldots \nu_{J_{2}}}\left(\Delta_{1}, \Delta_{2}, p_{X}\right)=\int d^{4} x d^{4} y \mathbf{e}^{-i \Delta_{1} x-i \Delta_{2} y} \\
& \cdot<0\left|T^{*} I^{\mu_{1} \ldots \mu_{J_{1}}}(x) I^{\nu_{1} \ldots \nu_{J_{1}}}(y) I_{\alpha_{1} \ldots \alpha_{J}}(0)\right| 0>
\end{aligned}
$$

where $I^{\mu_{1} \ldots \mu_{J}}$ is the current operator related to the hadronic spin- $J$ field operator,

$$
\left(\square+m_{J}^{2}\right) \Phi^{\mu_{1} \ldots \mu_{J}}(x)=I^{\mu_{1} \ldots \mu_{J}}(x) .
$$

The amplitude $T_{p p \rightarrow p X p}$ (Fig.1) is composed of vertices $T^{\mu_{1} \cdots \mu_{J_{1}}}, T^{\nu_{1} \cdots \nu_{J_{2}}}, F_{\alpha_{1} \ldots \alpha_{J}}^{\mu_{1} \ldots \mu_{J_{1}}, \nu_{1} \ldots \nu_{J_{2}}}$ and propagators $d(J, t) /\left(m^{2}(J)-t\right)$ which have the poles at

$$
m^{2}(J)-t=0 \text {, i.e. } J=\alpha(t) \text {, }
$$

after an appropriate analytic continuation of the signatured amplitudes in $J$. We assume that these poles, where $\alpha$ are Pomeron trajectories, give the dominant contribution at high energies after having taken the corresponding residues. Regge-cuts are generated by unitarization. 
For vertex functions $T_{1,2}$ we can obtain the following tensor decomposition:

$$
\begin{aligned}
& T^{\mu_{1} \ldots \mu_{J}}(p, \Delta)=T_{0}\left(\Delta^{2}\right) \sum_{n=0}^{\left[\frac{J}{2}\right]} Y_{J}^{n} \mathcal{T}_{00 J, n}^{\mu_{1} \ldots \mu_{J}}, \\
& Y_{J}^{n}=\frac{2^{n}(2(J-n)) ! J ! D_{p}^{2 n}}{(J-n) !(2 J) !} \\
& D_{p}^{\mu}=2 p^{\mu}-\Delta^{\mu}, D_{p}^{2}=4 m^{2}-\Delta^{2},
\end{aligned}
$$

that satisfies Rarita-Schwinger conditions:

$$
\begin{aligned}
& T^{\mu_{1} \ldots \mu_{i} \ldots \mu_{j} \ldots \mu_{J}}=T^{\mu_{1} \ldots \mu_{j} \ldots \mu_{i} \ldots \mu_{J}} \\
& \Delta_{\mu_{i}} T^{\mu_{1} \ldots \mu_{i} \ldots \mu_{J}}=0 \\
& g_{\mu_{i} \mu_{j}} T^{\mu_{1} \ldots \mu_{i} \ldots \mu_{j} \ldots \mu_{J}}=0
\end{aligned}
$$

Tensor structures $\mathcal{T}_{00 J, n}^{\mu_{1} \ldots \mu_{J}}$ satisfy only two conditions (15),(16) and consist of the elements $D_{p}^{\mu}$ and $G^{\mu \nu}$ :

$$
\begin{gathered}
G^{\mu \nu}=-g^{\mu \nu}+\frac{\Delta^{\mu} \Delta^{\nu}}{\Delta^{2}} \\
\mathcal{T}_{00 J, n}^{\mu_{1} \ldots \mu_{J}}=D_{p}^{\left(\mu_{1}\right.} \cdots D_{p}^{\mu_{J-2 n}} G^{\mu_{J-2 n+1} \mu_{J-2 n+2}} \cdots G^{\left.\mu_{J-1} \mu_{J}\right)}
\end{gathered}
$$

Let us assume the fusion of two particles with spins $J_{1}$ and $J_{2}$ into a particle with spin $J$. The general structure $F_{\alpha_{1} \ldots \alpha_{J}}^{\mu_{1} \ldots \mu_{J_{1}}, \nu_{1} \ldots \nu_{J_{2}}}\left(\Delta_{1}, \Delta_{2}, p_{X}\right)$ (see Fig.1) should satisfy conditions (15)-(17) on each group of indices. Since the contraction of the vertex with structures $T^{\mu_{1} \ldots \mu_{J_{1}}}\left(p_{1}, \Delta_{1}\right), T^{\nu_{1} \ldots \nu_{J_{2}}}\left(p_{2}, \Delta_{2}\right)$ and polarization tensor $e^{\alpha_{1} \ldots \alpha_{J}}\left(p_{X}\right)$ of the X particle leads to vanishing of some terms in $F$, the remainder can be constructed from

$$
p_{X}^{\mu_{i}}, p_{X}^{\nu_{j}}, \Delta_{1}^{\alpha_{k}}\left(\text { or } \Delta_{2}^{\alpha_{k}}\right), g^{\mu_{i} \nu_{j}}, g^{\mu_{i} \alpha_{k}}, g^{\nu_{j} \alpha_{k}}
$$

for tensors and additional terms

$$
\begin{aligned}
& \Lambda_{X}^{\mu_{i} \nu_{j} \alpha_{k}}=p_{X}^{\rho} \epsilon^{\rho \mu_{i} \nu_{j} \alpha_{k}}, \\
& \Lambda_{n}^{\mu_{i} \nu_{j} \alpha_{k}}=\Delta_{n}^{\rho} \epsilon^{\rho \mu_{i} \nu_{j} \alpha_{k}}, \\
& Q_{n}^{\lambda \kappa}=\Delta_{n}^{\rho} p_{X}^{\sigma} \epsilon^{\rho \sigma \lambda \kappa} \rightarrow \Delta_{1}^{\rho} \Delta_{2}^{\sigma} \epsilon^{\rho \sigma \lambda \kappa}, \\
& n=1 \text { or } 2,(\lambda \kappa)=\left(\mu_{i} \nu_{j}\right),\left(\mu_{i} \alpha_{k}\right),\left(\nu_{j} \alpha_{k}\right) \\
& i \leq J_{1}, j \leq J_{2}, k \leq J
\end{aligned}
$$

for pseudo-tensors [14]. Let $J_{1} \leq J_{2}$ and consider several cases.

- $J^{P}=0^{+}$

$$
\begin{aligned}
& F^{\mu_{1} \ldots \mu_{J_{1}}, \nu_{1} \ldots \nu_{J_{2}}}\left(\Delta_{1}, \Delta_{2}, p_{X}\right)=\sum_{k=0}^{J_{1}} f_{k} \cdot \\
& \cdot\left(p_{X}^{\mu_{1}} \cdots \cdots p_{X}^{\mu_{J_{1}-k}} \cdot g^{\mu_{J_{1}-k+1} \nu_{J_{2}-k+1}} \cdots \cdot g^{\mu_{J_{1}} \nu_{J_{2}}} \cdot p_{X}^{\nu_{J_{2}-k}} \cdots \cdots p_{X}^{\nu_{1}}\right) .
\end{aligned}
$$


After tensor contraction we obtain the expansion of the type

$$
\sum_{n=0}^{\left[\frac{J_{i}}{2}\right]} \frac{(-1)^{n} C_{J_{i}}^{n} C_{J_{i}}^{2 n}}{C_{2 J_{i}}^{2 n}}\left(\sqrt{\frac{\xi_{i}^{2}\left(m^{2}-t_{i} / 4\right)}{-t_{i}}} \frac{M_{X}^{2}-t_{1}-t_{2}}{M_{\perp}^{2}}\right)^{2 n} .
$$

In the kinematical region (3),(4) $\xi_{i}^{2} m^{2} /\left|t_{i}\right| \ll 1$ and we can keep only the first term of the expansion (25). The tensor product is given by

$$
\begin{aligned}
& T^{J_{1}}\left(p_{1}, \Delta_{1}\right) \otimes F^{J_{1}, J_{2}, 0^{+}}\left(\Delta_{1}, \Delta_{2}\right) \otimes T^{J_{2}}\left(p_{2}, \Delta_{2}\right) \sim \\
& \sim s_{1}^{J_{1}} s_{2}^{J_{2}} \sum_{k=0}^{J_{1}} \frac{f_{k} 2^{k}}{M_{\perp}^{2 k}} .
\end{aligned}
$$

- $J^{P}=0^{-}$

$$
\begin{aligned}
& F^{\mu_{1} \ldots \mu_{J_{1}}, \nu_{1} \ldots \nu_{J_{2}}}\left(\Delta_{1}, \Delta_{2}, p_{X}\right)=Q_{n}^{\mu_{1} \nu_{1}} \sum_{k=0}^{J_{1}-1} f_{k} \cdot \\
& \cdot\left(p_{X}^{\mu_{2}} \cdots \cdots p_{X}^{\mu_{J_{1}-k}} \cdot g^{\mu_{J_{1}-k+1} \nu_{J_{2}-k+1}} \cdots \cdots g^{\mu_{J_{1}} \nu_{J_{2}}} \cdot p_{X}^{\nu_{J_{2}-k}} \cdots \cdots p_{X}^{\nu_{2}}\right) \\
& T^{J_{1}}\left(p_{1}, \Delta_{1}\right) \otimes F^{J_{1}, J_{2}, 0^{-}}\left(\Delta_{1}, \Delta_{2}\right) \otimes T^{J_{2}}\left(p_{2}, \Delta_{2}\right) \sim \\
& \sim 4 Q_{n}^{\mu_{1} \nu_{1}} p_{1}^{\mu_{1}} p_{2}^{\nu_{1}} s_{1}^{J_{1}-1} s_{2}^{J_{2}-1} \sum_{k=0}^{J_{1}-1} \frac{f_{k} 2^{k}}{M_{\perp}^{2 k}} \\
& \simeq\left[\boldsymbol{\Delta}_{1} \times \boldsymbol{\Delta}_{2}\right] \cdot s_{1}^{J_{1}} s_{2}^{J_{2}} \sum_{k=0}^{J_{1}-1} \frac{f_{k} 2^{k+1}}{M_{\perp}^{2 k+2}}
\end{aligned}
$$

- $J^{P}=1^{-}$

$$
\begin{aligned}
& F^{\mu_{1} \ldots \mu_{J_{1}}, \nu_{1} \ldots \nu_{J_{2}}, \alpha}\left(\Delta_{1}, \Delta_{2}, p_{X}\right)=g^{\alpha \mu_{1}} \sum_{k=0}^{J_{1}-1} f_{k} \cdot \\
& \cdot\left(p_{X}^{\mu_{2}} \cdots \cdots p_{X}^{\mu_{J_{1}-k}} \cdot g^{\mu_{J_{1}-k+1} \nu_{J_{2}-k+1}} \cdots \cdots g^{\mu_{J_{1}} \nu_{J_{2}}} \cdot p_{X}^{\nu_{J_{2}-k}} \cdots \cdots p_{X}^{\nu_{1}}\right)+ \\
& +g^{\alpha \nu_{1}} \sum_{k=0}^{J_{1}} f_{J_{1}+k} \cdot \\
& \cdot\left(p_{X}^{\mu_{1}} \cdots \cdots p_{X}^{\mu_{J_{1}-k}} \cdot g^{\mu_{J_{1}-k+1} \nu_{J_{2}-k+1}} \cdots \cdots g^{\mu_{J_{1}} \nu_{J_{2}}} \cdot p_{X}^{\nu_{J_{2}-k}} \cdots \cdot p_{X}^{\nu_{2}}\right)+ \\
& +\Delta_{n}^{\alpha} \sum_{k=0}^{J_{1}} f_{2 J_{1}+k+1} \cdot \\
& \cdot\left(p_{X}^{\mu_{1}} \cdots \cdots p_{X}^{\mu_{J_{1}-k}} \cdot g^{\mu_{J_{1}-k+1} \nu_{J_{2}-k+1}} \cdots \cdots g^{\mu_{J_{1}} \nu_{J_{2}}} \cdot p_{X}^{\nu_{J_{2}-k}} \cdots \cdots p_{X}^{\nu_{1}}\right)
\end{aligned}
$$




$$
\begin{aligned}
& T^{J_{1}}\left(p_{1}, \Delta_{1}\right) \otimes F^{J_{1}, J_{2}, 1^{-}}\left(\Delta_{1}, \Delta_{2}\right) \otimes T^{J_{2}}\left(p_{2}, \Delta_{2}\right) \sim \\
& \sim s_{1}^{J_{1}} s_{2}^{J_{2}}\left[\frac{2 p_{1}^{\alpha}}{s_{1}} \sum_{k=0}^{J_{1}-1} \frac{f_{k} 2^{k}}{M_{\perp}^{2 k}}+\right. \\
& +\frac{2 p_{2}^{\alpha}}{s_{2}} \sum_{k=0}^{J_{1}} \frac{f_{J_{1}+k} 2^{k}}{M_{\perp}^{2 k}}+ \\
& \left.+\Delta_{n}^{\alpha} \sum_{k=0}^{J_{1}} \frac{f_{2 J_{1}+1+k} 2^{k}}{M_{\perp}^{2 k}}\right] .
\end{aligned}
$$

It is easy to show from the general form of tensor decompositions, that after reggeization procedure one obtains the structure of the amplitude, which is similar to the special case $J_{1}=J_{2}=1$. In this case it is convenient to use the following bosesymmetric form of the tensor

$$
\begin{aligned}
& F^{\mu \nu, \alpha}\left(\Delta_{1}, \Delta_{2}, p_{X}\right)=f_{0} g^{\alpha \mu} \Delta_{1}^{\nu}+\bar{f}_{0} g^{\alpha \nu} \Delta_{2}^{\mu}+ \\
& +\left(f_{1} \Delta_{1}^{\alpha}+\bar{f}_{1} \Delta_{2}^{\alpha}\right) \Delta_{2}^{\mu} \Delta_{1}^{\nu}+ \\
& +\left(f_{2} \Delta_{1}^{\alpha}+\bar{f}_{2} \Delta_{2}^{\alpha}\right) g^{\mu \nu}
\end{aligned}
$$

and the tensor product

$$
\begin{aligned}
& T^{J_{1}}\left(p_{1}, \Delta_{1}\right) \otimes F^{J_{1}, J_{2}, 1^{-}}\left(\Delta_{1}, \Delta_{2}\right) \otimes T^{J_{2}}\left(p_{2}, \Delta_{2}\right) \sim \\
& \sim s_{1}^{J_{1}} s_{2}^{J_{2}}\left[\frac{2 p_{1}^{\alpha}}{s_{1}} f_{0}+\frac{2 p_{2}^{\alpha}}{s_{2}} \overline{f_{0}}+\left(f_{1}+\frac{f_{2}}{M_{\perp}^{2}}\right) \Delta_{1}^{\alpha}+\right. \\
& \left.+\left(\bar{f}_{1}+\frac{\bar{f}_{2}}{M_{\perp}^{2}}\right) \Delta_{2}^{\alpha}\right],
\end{aligned}
$$

where $\bar{f}\left(t_{1}, t_{2}\right)=f\left(t_{2}, t_{1}\right)$.

- $J^{P}=1^{+}$

$$
\begin{aligned}
& F^{\mu_{1} \ldots \mu_{J_{1}}, \nu_{1} \ldots \nu_{J_{2}}, \alpha}\left(\Delta_{1}, \Delta_{2}, p_{X}\right)=\Lambda_{X}^{\mu_{1} \nu_{1} \alpha} \sum_{k=0}^{J_{1}-1} f_{k} \cdot \\
& \cdot\left(p_{X}^{\mu_{2}} \cdots \cdots p_{X}^{\mu_{J_{1}-k}} \cdot g^{\mu_{J_{1}-k+1} \nu_{J_{2}-k+1}} \cdots g^{\mu_{J_{1} \nu_{J_{2}}}} \cdot p_{X}^{\nu_{J_{2}-k}} \cdots \cdots p_{X}^{\nu_{2}}\right)+ \\
& +\Lambda_{n}^{\mu_{1} \nu_{1} \alpha} \sum_{k=0}^{J_{1}-1} f_{J_{1}+k} \cdot \\
& \cdot\left(p_{X}^{\mu_{2}} \cdots p_{X}^{\mu_{J_{1}-k}} \cdot g^{\mu_{J_{1}-k+1} \nu_{J_{2}-k+1}} \cdots \cdot g^{\mu_{J_{1}} \nu_{J_{2}}} \cdot p_{X}^{\nu_{J_{2}-k}} \cdots p_{X}^{\nu_{2}}\right)+ \\
& +Q_{n}^{\nu_{1} \alpha} f_{2 J_{1}}\left(g^{\mu_{1} \nu_{2}} \cdots \cdots g^{\mu_{J_{1}} \nu_{J_{1}+1}} \cdot p_{X}^{\nu_{J_{1}+2}} \cdots \cdots p_{X}^{\nu_{J_{2}}}\right)+ \\
& +Q_{n}^{\mu_{1} \alpha} \sum_{k=0}^{J_{1}-1} f_{2 J_{1}+k+1} \cdot \\
& \cdot\left(p_{X}^{\mu_{2}} \cdots \cdots p_{X}^{\mu_{J_{1}-k}} \cdot g^{\mu_{J_{1}-k+1} \nu_{J_{2}-k+1}} \cdots \cdots g^{\mu_{J_{1}} \nu_{J_{2}}} \cdot p_{X}^{\nu_{J_{2}-k}} \cdots p_{X}^{\nu_{1}}\right)
\end{aligned}
$$




$$
\begin{aligned}
& T^{J_{1}}\left(p_{1}, \Delta_{1}\right) \otimes F^{J_{1}, J_{2}, 1^{+}}\left(\Delta_{1}, \Delta_{2}\right) \otimes T^{J_{2}}\left(p_{2}, \Delta_{2}\right) \sim \\
& \sim s_{1}^{J_{1}} s_{2}^{J_{2}}\left[\frac{2 p_{1}^{\mu_{1}} p_{2}^{\nu_{1}} p_{X}^{\rho} \epsilon^{\rho \alpha \mu_{1} \nu_{1}}}{s} \sum_{k=0}^{J_{1}-1} \frac{f_{k} 2^{k+1}}{M_{\perp}^{2 k+2}}+\right. \\
& +\frac{2 p_{1}^{\mu_{1}} p_{2}^{\nu_{1}} \Delta_{n}^{\rho} \epsilon^{\rho \alpha \mu_{1} \nu_{1}}}{s} \sum_{k=0}^{J_{1}-1} \frac{f_{J_{1}+k} 2^{k+1}}{M_{\perp}^{2 k+2}}+ \\
& +\frac{2 p_{2}^{\nu_{1}} p_{X}^{\rho} \Delta_{n}^{\sigma} \epsilon^{\rho \sigma \alpha \nu_{1}}}{s_{2}} \frac{f_{2 J_{1}} 2^{J_{1}}}{M_{\perp}^{2 J_{1}}}+ \\
& \left.+\frac{2 p_{1}^{\mu_{1}} p_{X}^{\rho} \Delta_{n}^{\sigma} \epsilon^{\rho \sigma \alpha \mu_{1}}}{s_{1}} \sum_{k=0}^{J_{1}-1} \frac{f_{2 J_{1}+1+k} 2^{k}}{M_{\perp}^{2 k}}\right] .
\end{aligned}
$$

For $J_{1}=J_{2}=1$

$$
\begin{aligned}
& T^{J_{1}}\left(p_{1}, \Delta_{1}\right) \otimes F^{J_{1}, J_{2}, 1^{+}}\left(\Delta_{1}, \Delta_{2}\right) \otimes T^{J_{2}}\left(p_{2}, \Delta_{2}\right) \sim \\
& \sim s_{1}^{J_{1}} s_{2}^{J_{2}}\left[\frac{4 p_{1}^{\sigma} p_{2}^{\lambda}\left(f_{0} \Delta_{1}^{\rho}-\bar{f}_{0} \Delta_{2}^{\rho}\right)}{s M_{\perp}^{2}}+2 \Delta_{1}^{\rho} \Delta_{2}^{\sigma}\left(\frac{f_{1} p_{1}^{\lambda}}{s_{1}}-\frac{\bar{f}_{1} p_{2}^{\lambda}}{s_{2}}\right)\right] \epsilon^{\rho \sigma \lambda \alpha}= \\
& =2 s_{1}^{J_{1}} s_{2}^{J_{2}}\left[p_{1}^{\lambda} P_{2}^{\rho} \Delta_{1}^{\sigma}+p_{2}^{\lambda} P_{1}^{\rho} \Delta_{2}^{\sigma}\right] \epsilon^{\rho \sigma \lambda \alpha}, \\
& P_{1}=-\frac{\bar{f}_{1}}{s_{2}} \Delta_{1}+\frac{\bar{f}_{0}}{s M_{\perp}^{2}} 2 p_{1}, \\
& P_{2}=-\frac{f_{1}}{s_{1}} \Delta_{2}+\frac{f_{0}}{s M_{\perp}^{2}} 2 p_{2} .
\end{aligned}
$$

- $J^{P}=2^{+}$. For simplicity we consider only the case $J_{1}=J_{2}=1$.

$$
\begin{aligned}
& F^{\mu \nu, \alpha_{1} \alpha_{2}}\left(\Delta_{1}, \Delta_{2}, p_{X}\right)=f_{0} g^{\alpha_{1} \mu} g^{\alpha_{2} \nu}+ \\
& +f_{1} g^{\alpha_{1} \mu} \Delta_{1}^{\alpha_{2}} \Delta_{1}^{\nu}+\bar{f}_{1} g^{\alpha_{1} \nu} \Delta_{2}^{\alpha_{2}} \Delta_{2}^{\mu}+\left(f_{2} \Delta_{1}^{\alpha_{1}} \Delta_{1}^{\alpha_{2}}+\bar{f}_{2} \Delta_{2}^{\alpha_{1}} \Delta_{2}^{\alpha_{2}}\right) g^{\mu \nu}+ \\
& +\left(f_{3} \Delta_{1}^{\alpha_{1}} \Delta_{1}^{\alpha_{2}}+\bar{f}_{3} \Delta_{2}^{\alpha_{1}} \Delta_{2}^{\alpha_{2}}\right) \Delta_{2}^{\mu} \Delta_{1}^{\nu}, \\
& \quad T^{J_{1}}\left(p_{1}, \Delta_{1}\right) \otimes F^{J_{1}, J_{2}, 2^{+}}\left(\Delta_{1}, \Delta_{2}\right) \otimes T^{J_{2}}\left(p_{2}, \Delta_{2}\right) \sim \\
& \quad \sim s_{1}^{J_{1}} s_{2}^{J_{2}}\left[f_{0} \frac{4 p_{1}^{\alpha_{1}} p_{2}^{\alpha_{2}}}{s M_{\perp}^{2}}+f_{1} \frac{2 p_{1}^{\alpha_{1}} \Delta_{1}^{\alpha_{2}}}{s_{1}}+\bar{f}_{1} \frac{2 p_{2}^{\alpha_{1}} \Delta_{2}^{\alpha_{2}}}{s_{2}}+\right. \\
& \left.\quad+\frac{2\left(f_{2} \Delta_{1}^{\alpha_{1}} \Delta_{1}^{\alpha_{2}}+\bar{f}_{2} \Delta_{2}^{\alpha_{1}} \Delta_{2}^{\alpha_{2}}\right)}{M_{\perp}^{2}}+\left(f_{3} \Delta_{1}^{\alpha_{1}} \Delta_{1}^{\alpha_{2}}+\bar{f}_{3} \Delta_{2}^{\alpha_{1}} \Delta_{2}^{\alpha_{2}}\right)\right] .
\end{aligned}
$$

Everywhere in the above expressions $f_{k}=f_{k}^{J^{P}}\left(t_{1}, t_{2}, M_{X}^{2}\right)$.

\section{Azimuthal angle dependence.}

In this section we will obtain the general structure of the azimuthal angle dependence for different $J^{P}$ states in the tensor current picture. If we assume that the dominant 
contribution is given by Regge poles $\alpha_{1}\left(t_{1}\right)$ and $\alpha_{2}\left(t_{2}\right)$ then the amplitude of the process can be written in the following form

$$
T_{p p \rightarrow p X p} \sim \eta\left(\alpha_{1}\left(t_{1}\right)\right) \eta\left(\alpha_{2}\left(t_{2}\right)\right)\left[T^{J_{1}} \otimes F^{J_{1}, J_{2}, J^{P}} \otimes T^{J_{2}}\right]_{\substack{J_{1} \rightarrow \alpha_{1} \\ J_{2} \rightarrow \alpha_{2}}}
$$

where $J_{i} \rightarrow \alpha_{i}$ means the usual procedure of the analytical continuation to the complex $J$-plane and taking residues at Regge poles, $\eta\left(\alpha_{i}\right)$ are signature factors.

In the most important case, when the main contribution is given by one Pomeron trajectory, $\alpha_{1}=\alpha_{2}=\alpha_{\mathbb{P}}(0)$ and the "bare" amplitude squared is proportional to following expressions:

- $J^{P}=0^{+}$

$$
\left|T_{p p \rightarrow p X p}\right|^{2} \sim\left(M_{\perp}^{2}\right)^{2\left(\alpha_{\mathbb{P}}(0)-1\right)}\left(f_{0} M_{\perp}^{2}+2 f_{1}\right)^{2}
$$

- $J^{P}=0^{-}$

$$
\left|T_{p p \rightarrow p X p}\right|^{2} \sim\left(M_{\perp}^{2}\right)^{2\left(\alpha_{\mathbb{P}}(0)-1\right)} f_{0} t_{1} t_{2} \sin ^{2} \phi_{0}
$$

- $J^{P}=1^{-}$. In this case we assume the existence of a C-odd vacuum trajectory, "Odderon", $\alpha_{\mathbb{O}}(t)$.

$$
\begin{aligned}
& \left|T_{p p \rightarrow p X p}\right|^{2} \sim\left(M_{\perp}^{2}\right)^{\alpha_{\mathbb{P}}(0)+\alpha_{\mathbb{O}}(0)-2}\left(\mathcal{F}_{0} M_{\perp}^{4}+\mathcal{F}_{1} M_{\perp}^{2}+\mathcal{F}_{2}\right) \\
& \mathcal{F}_{0}=\frac{f_{0}^{S^{2}}}{M_{X}^{2}}+f_{0}^{A} f_{1}^{A}+\frac{\left(t_{1}-t_{2}\right) f_{1}^{A} f_{0}^{S}}{M_{X}^{2}}+\frac{f_{1}^{A^{2}} \lambda}{4 M_{X}^{2}} \\
& \mathcal{F}_{1}=f_{0}^{A^{2}}-f_{0}^{S^{2}}-f_{0}^{A} f_{2}^{A}+\frac{\left(t_{1}-t_{2}\right) f_{2}^{A} f_{0}^{S}}{M_{X}^{2}}+\frac{f_{1}^{A} f_{2}^{A} \lambda}{2 M_{X}^{2}} \\
& \mathcal{F}_{2}=\frac{f_{2}^{A^{2}} \lambda}{4 M_{X}^{2}} \\
& \lambda=\lambda\left(M_{X}^{2}, t_{1}, t_{2}\right)=M_{X}^{4}+t_{1}^{2}+t_{2}^{2}-2 M_{X}^{2} t_{1}-2 M_{X}^{2} t_{2}-2 t_{1} t_{2} \\
& f_{k}^{S}=f_{k}+\bar{f}_{k}, f_{k}^{A}=f_{k}-\bar{f}_{k} .
\end{aligned}
$$

- $J^{P}=1^{+}$

$$
\begin{aligned}
\left|T_{p p \rightarrow p X p}\right|^{2} & \sim\left(M_{\perp}^{2}\right)^{2\left(\alpha_{\mathbb{P}}(0)-1\right)}\left(\mathcal{F}_{0} M_{\perp}^{4}+\mathcal{F}_{1} t_{1} t_{2} \sin ^{2} \phi_{0}+\mathcal{F}_{2}\right) \\
\mathcal{F}_{0} & =\left(f_{1} \Delta_{1}-\bar{f}_{1} \Delta_{2}\right)^{2} \\
\mathcal{F}_{1} & =\frac{2\left(s_{2} f_{1}-s_{1} \bar{f}_{1}\right)^{2}}{s}+4 M_{\perp}^{2} f_{1} \bar{f}_{1}-\frac{4\left(f_{0}+\bar{f}_{0}\right)^{2}}{M_{X}^{2}} \\
\mathcal{F}_{2} & =4\left(f_{0} \boldsymbol{\Delta}_{1}-\bar{f}_{0} \boldsymbol{\Delta}_{2}\right)^{2}
\end{aligned}
$$

- $J^{P}=2^{+}$

$$
\left|T_{p p \rightarrow p X p}\right|^{2} \sim\left(M_{\perp}^{2}\right)^{2\left(\alpha_{\mathbb{P}}(0)-1\right)}\left(\mathcal{F}_{0} M_{\perp}^{4}+\mathcal{F}_{1} M_{\perp}^{2}+\mathcal{F}_{2}\right)
$$




$$
\begin{aligned}
& \mathcal{F}_{0}=\frac{f_{1}^{S^{2}}-12 f_{0} f_{3}^{S}}{24}+\frac{f_{1}^{S}\left(-4 f_{0}+f_{1}^{A}\left(t_{1}-t_{2}\right)+2 \lambda f_{3}^{S}\right)}{12 M_{X}^{2}}+ \\
& +\frac{1}{24 M_{X}^{4}}\left[16 f_{0}^{2}+4 f_{0}\left(4 f_{1}^{A}\left(t_{1}-t_{2}\right)+f_{3}^{S}\left(3\left(t_{1}-t_{2}\right)^{2}-\lambda\right)\right)+\right. \\
& \left.+4 f_{3}^{S} f_{1}^{A}\left(t_{1}-t_{2}\right) \lambda+f_{3}^{S^{2}} \lambda+f_{1}^{A^{2}}\left(\left(t_{1}-t_{2}\right)^{2}+3 \lambda\right)\right] \\
& \mathcal{F}_{1}=-\frac{1}{3} f_{0}\left(f_{1}^{S}+3 f_{2}^{S}\right)+ \\
& +\frac{f_{2}^{S}}{6 M_{X}^{4}}\left[\lambda^{2} f_{3}^{S}+2 \lambda\left(-f_{0}+f_{1}^{A}\left(t_{1}-t_{2}\right)\right)+6 f_{0}\left(t_{1}-t_{2}\right)^{2}\right]+ \\
& +\frac{1}{6 M_{X}^{2}}\left[\lambda\left(2 f_{1}^{S} f_{2}^{S}+2 f_{0} f_{3}^{S}+3\left(f_{1}^{S^{2}}-f_{1}^{A^{2}}\right) / 4\right)-4 f_{0}^{2}-2 f_{0} f_{1}^{A}\left(t_{1}-t_{2}\right)\right], \\
& \mathcal{F}_{2}=\frac{\left(2 f_{0}+f_{2}^{S} \lambda / M_{X}^{2}\right)^{2}}{6} .
\end{aligned}
$$

Similar formulae for the differential cross-sections were obtained by other authors. In Ref. [15] results were obtained from the assumption that the Pomeron acts as a $1^{+}$ conserved or nonconserved current. It was shown in Ref. [16] and with more detailed investigations in Ref. [17] that the same result follows from the simple Regge behaviour of helicity amplitudes. Experimental data are in good agreement with these predictions.

A good description of those data in the framework of our approach (with account of the absorbtion) is obtained (Figs. 3,4).

In addition to the present description there are some models of "glueball" based on the "instanton" dynamics [18].

\section{WA102 and predictions for LHC.}

It is important to stress the fact, that at WA102 energies absorbtive effects are not so significant, and azimuthal angle dependence looks like the "bare" one. We can use this fact to simplify the fitting procedure, that has been already done by WA102 collaboration. Only at large values of $\mathrm{dP} \mathrm{P}_{\perp}=\left|\boldsymbol{\Delta}_{1}-\boldsymbol{\Delta}_{2}\right|$ the process of "soft" rescattering can change the picture violently (see Fig. 3d).

In Figs. 3,4 we show the data from WA102 [7] and our curves for "bare" and unitarized amplitudes. One can see that all the features of the average $\phi_{0}$-dependence are consistent with the data. It makes possible to predict azimuthal angle behaviour at higher energies and use these predictions as a spin-parity analyser.

The main property is that unitarization adds up to the distortion of the "bare" crosssection towards small angles and to the reduction of its value. The difference is more significant at LHC than at WA102 energies, and we should take it into account necessarily. "Soft" survival probability is $0.25 \rightarrow 0.3$ for WA102 and $0.05 \rightarrow 0.1$ for LHC. It depends on mass $M_{X}$ and kinematical cuts.

Features concerning each particle are the same as was mentioned in Ref. [15]:

- for $\eta^{\prime}$ mesons the kinematical distortion because of the different reference frame is 
totally compensated by unitarization at WA102 energies (Fig. 3a), and for LHC the peak in the cross-section is shifted to $65^{\circ}$ (Fig. 5a).

- for $\mathrm{f}_{1}(1285)$ we have almost "flat" distribution at large values of $\left|t_{1}-t_{2}\right|$ (Fig. 3d), since in the simplest case its cross-section is proportional to $\mathrm{dP}_{\perp}^{2}=\left(\boldsymbol{\Delta}_{1}-\boldsymbol{\Delta}_{2}\right)^{2}$. For LHC we obtain more stronger suppression for large angles (Fig. 5d).

- the difference between $0^{++} q \bar{q}$ and non- $q \bar{q}$ states at WA102 (Figs. 4a,b correspondingly) changes due to unitarization effects. For $q \bar{q}$ azimuthal angle dependence becomes almost "flat" (Fig. 6b), and for non- $q \bar{q}$ mesons we see the shrinkage of the peak at $\phi_{0}=0^{\circ}$. The same is valid for $2^{++}$mesons.

For low mass particles, which can be produced in the EDDE, total cross-sections are estimated to be of the order $1 \div 30 \mu b$ at LHC. Cross-sections for "glueball" candidates $\mathrm{f}_{0}(1500)$ and $\mathrm{f}_{2}(1950)$ are about $30 \mu b$ (depend on the LHC kinematics and may be larger) and effective slope is 10 . Typical values of $\xi$ are of the order $M_{X} / \sqrt{s} \sim 10^{-4}$.

It is possible to apply the method to large mass particles. In Ref. [19] it was argued that a heavy glueball ("knot") $1^{--}$with mass near $50 \mathrm{GeV}$ may exist. One can see from (40)-(48) that in this case $\phi_{0}$ dependence is simply defined by unitarization only, since $M_{\perp}^{2} \simeq M_{X}^{2}=$ const. In this case we have a good tool to check models for "soft" rescattering. Examples are depicted in Fig.7.

\section{Conclusions}

Detailed investigations of the azimuthal angle dependence in the EDDE can help to solve several important problems:

- to check different models for "soft" processes and to study the real pattern of the interaction.

- to understand the difference in the dynamics of production of $q \bar{q}$ and non- $q \bar{q}$ states and their possible filtering.

- to determine the quantum numbers of new produced particles.

Experimental possibilities of angular distributions measurements should be studied in further Monte Carlo simulations.

\section{Aknowledgements}

The work is supported by grants SNRS-PICS-2910 and RFBR-04-02-17299.

\section{References}

[1] T.W. Kibble, Proc. Roy. Soc. 244 (1958) 355.

A.A. Logunov and A.N. Tavkhelidze, Nucl. Phys. 8 (1958) 374. 
[2] K.A. Ter-Martirosyan, Nucl. Phys. 68 (1964) 591;

K.G. Boreskov, Yad. Fiz. V.8 S.4 (1968) 796.

[3] V.A. Petrov and R.A. Ryutin, JHEP 0408 (2004) 013;

V.A. Petrov and R.A. Ryutin, Eur. Phys. Journ. C 36 (2004) 509.

[4] F.E. Close, A. Kirk, Eur. Phys. J. C 21 (2001) 531, hep-ph/0103173.

[5] S.S. Gershtein and A.A. Logunov, Sov. J. Nucl. Phys. 39 (1984) 1514.

[6] Yu.D. Prokoshkin, IHEP preprint 88-40. Serpukhov, 1988.

[7] WA102 Coll., Phys. Lett. B 427 (1998) 398, hep-ex/9803029;

Phys. Lett. B 467 (1999) 165, hep-ex/9909013;

Phys. Lett. B 474 (2000) 423, hep-ex/0001017;

[8] WA102 Coll., Phys. Lett. B 462 (1999) 462, hep-ex/9907055.

[9] A. Kirk, Phys. Lett. B 489 (2000) 29, hep-ph/0008053.

[10] F.E. Close, Phys. Lett. B 419 (1998) 387, hep-ph/9710450;

WA102 Coll., A. Kirk et al., hep-ph/9810221;

F.E. Close, A. Kirk, Phys. Lett. B 397 (1997) 333, hep-ph/9701222;

F.E. Close, A. Kirk, G.A. Schuler, Phys. Lett. B 477 (2000) 13, hep-ph/0001158.

[11] A. Berera and J. C. Collins, Nucl. Phys. B 474 (1996) 183.

[12] J.-R. Cudell and O. F. Hernandez, Nucl. Phys. B 471 (1996) 471.

[13] V.A. Petrov and A. V. Prokudin, Eur. Phys. J. C 23 (2002)135.

[14] Jie-Jie Zhu, Mu-Lin Yan, hep-ph/9903349.

[15] F.E. Close, G.A. Schuller, Phys. Lett. B 458(1999) 127, hep-ph/9902243;

F.E. Close, G.A. Schuller, Phys. Lett. B 464 (1999) 279, hep-ph/9905305.

[16] A.B. Kaidalov, V.A. Khoze, A.D. Martin and M.G. Ryskin, Eur. Phys. J. C 31 (2003) 387, hep-ph/0307064.

[17] V.A. Khoze, A.D. Martin and M.G. Ryskin, Eur. Phys. J. C 24 (2002) 581, hep$\mathrm{ph} / 0203122$.

[18] E. Shuryak, I. Zahed, Phys. Rev. D 68 (2003) 034001, hep-ph/0302231;

N. Kochelev, hep-ph/9902203

[19] Y.M. Cho, hep-th/0406004. 


\section{Figure captions}

Fig. 1: The process $p+p \rightarrow p+X+p$. Absorbtion in the initial and final pp-channels is not shown.

Fig. 2: The full unitarization of the process $p+p \rightarrow p+X+p$.

Fig. 3: Experimental data from WA102. Dashed curve represents "bare" cross-section and solid one is the unitarized result. a) $\eta^{\prime}, 0^{-+}$; b) $f_{1}(1285), 1^{++}$, all $t_{i}$; c) $f_{1}(1285)$, $\left|t_{1}-t_{2}\right|<0.2 \mathrm{GeV}^{2}$; d) $f_{1}(1285),\left|t_{1}-t_{2}\right|>0.4 \mathrm{GeV}^{2}$;

Fig. 4: Experimental data from WA102, averaged over all measured $t_{i}$ values. Dashed curve represents "bare" cross-section and solid one is the unitarized result. a) $f_{0}(980), 0^{++}$; b) $f_{0}(1500), 0^{++}$; c) $f_{2}(1270), 2^{++}$; d) $f_{2}(1950), 2^{++}$;

Fig. 5: Results for LHC energies. a) $\eta^{\prime}, 0^{-+}$; b) $f_{1}(1285), 1^{++}$, all $t_{i}$; c) $f_{1}(1285)$, $\left|t_{1}-t_{2}\right|<0.1 \mathrm{GeV}^{2}$; d) $f_{1}(1285),\left|t_{1}-t_{2}\right|>0.2 \mathrm{GeV}^{2}$

Fig. 6: Results for LHC energies. a) $f_{0}(980), 0^{++}$; b) $f_{0}(1500), 0^{++}$; c) $f_{2}(1270), 2^{++}$; d) $f_{2}(1950), 2^{++}$;

Fig. 7: Examples of azimuthal angle dependence for large mass states. a) $1^{--}, M_{X}=$ $50 \mathrm{GeV}$; b) $0^{-+}, M_{X}=50 \mathrm{GeV}$; 


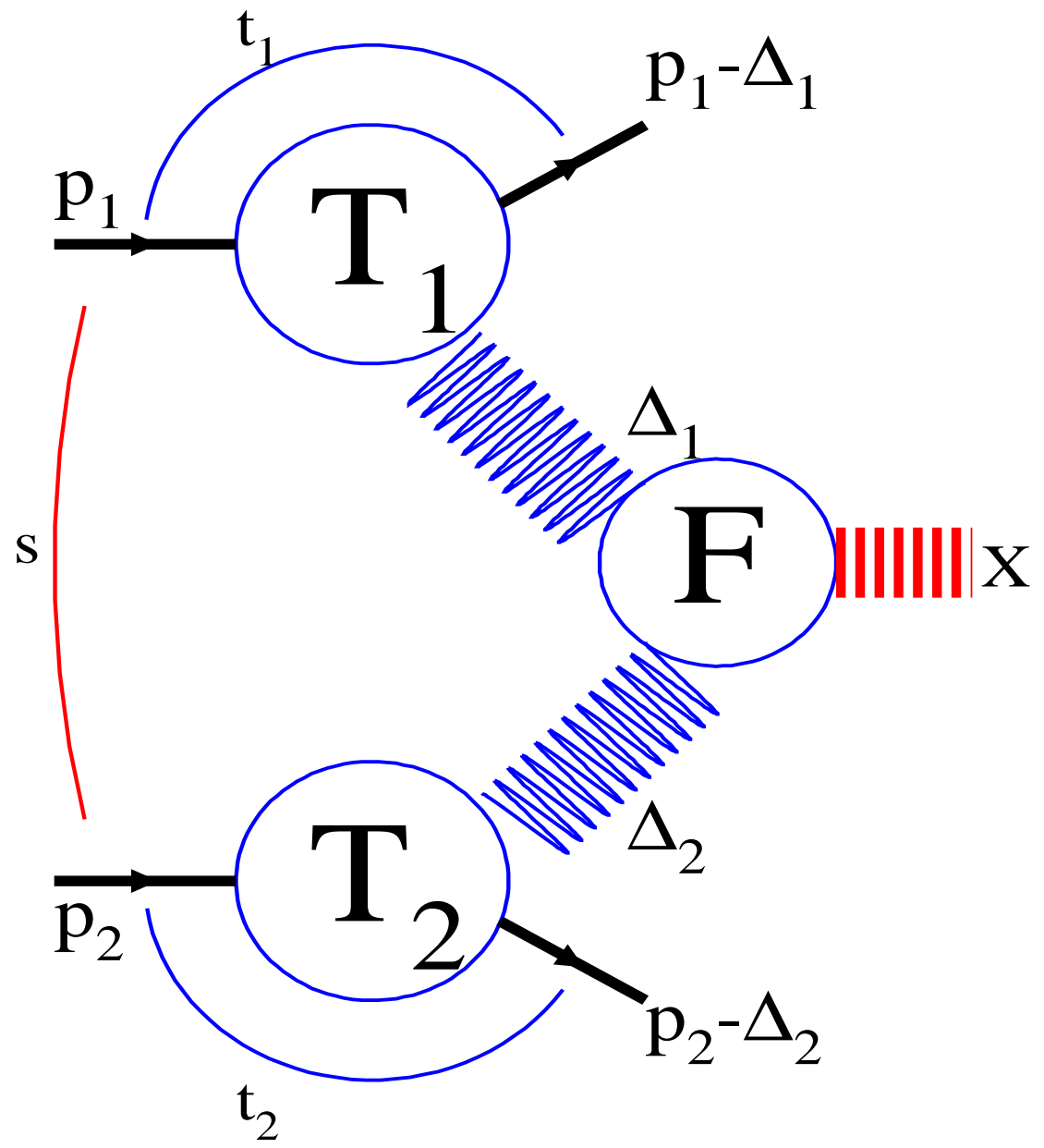

Figure 1: 


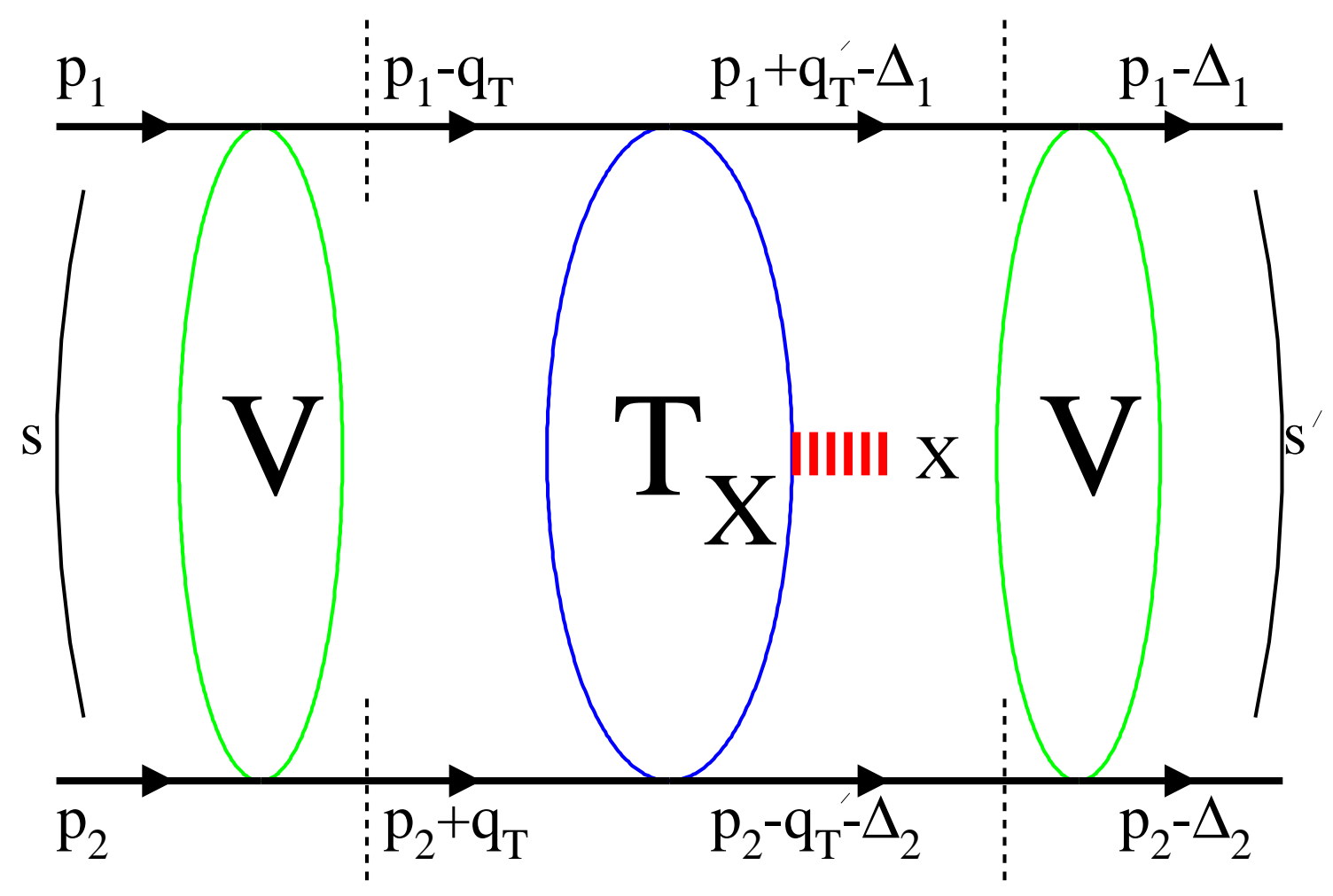

Figure 2: 
$d \sigma_{p p \rightarrow p X p} / d \phi / \sigma_{p p \rightarrow p X p}$

a)

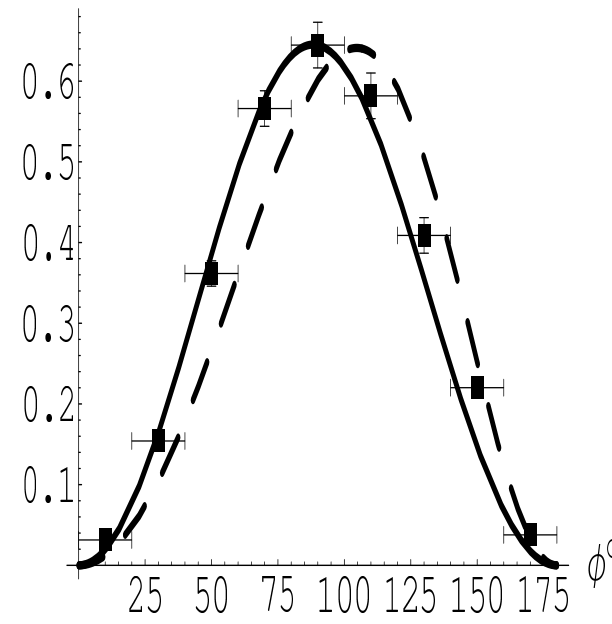

$d \sigma_{\mathrm{pp} \rightarrow \mathrm{pXp}} / d \phi / \sigma_{\mathrm{pp} \rightarrow \mathrm{p} \mathrm{Xp}}$

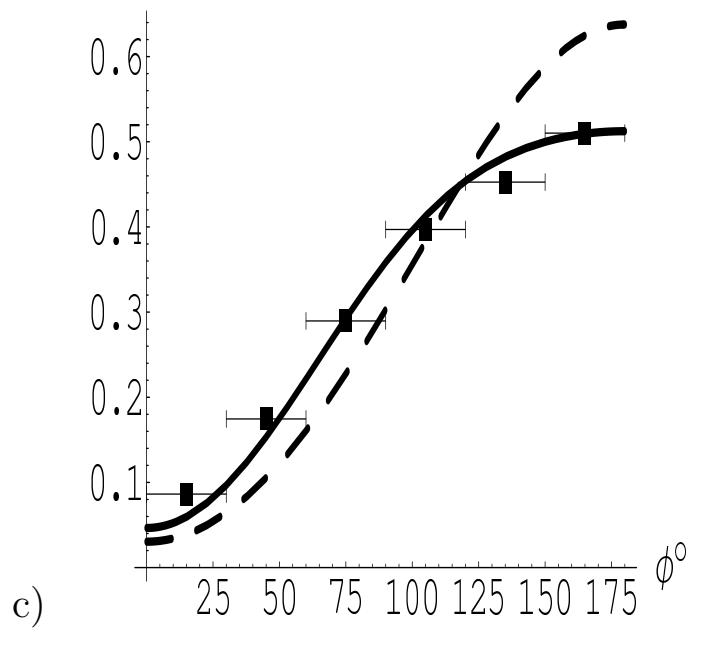

$d \sigma_{p p \rightarrow p x p} / d \phi / \sigma_{p p \rightarrow p x p}$

b)

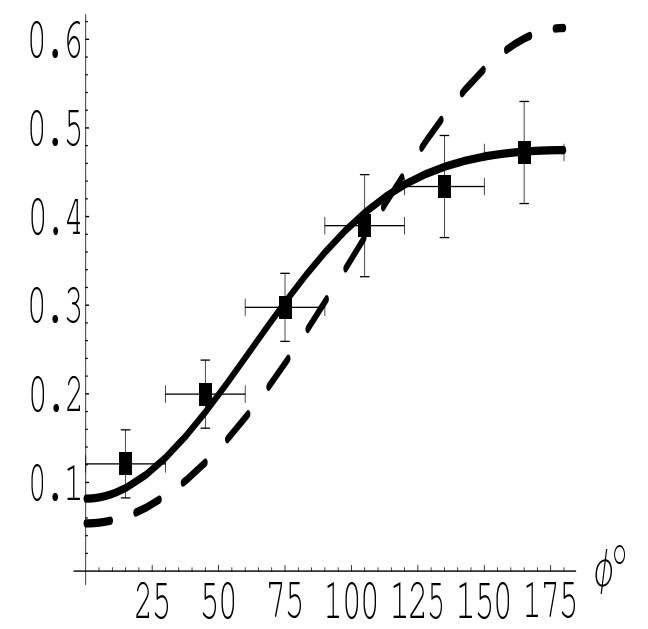

$d \sigma_{p p \rightarrow p X p} / d \phi / \sigma_{p p \rightarrow p x p}$

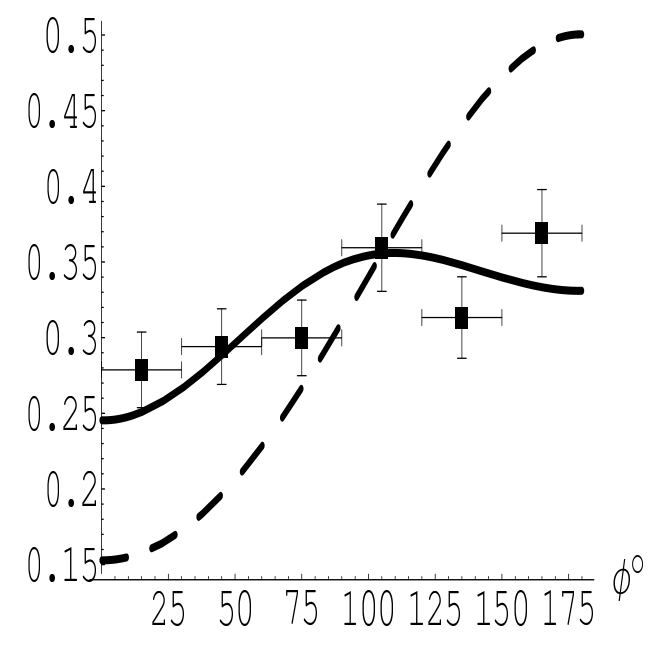

Figure 3: 
$d \sigma_{p p \rightarrow p X p} / d \phi / \sigma_{p p p \rightarrow p x p}$

a)

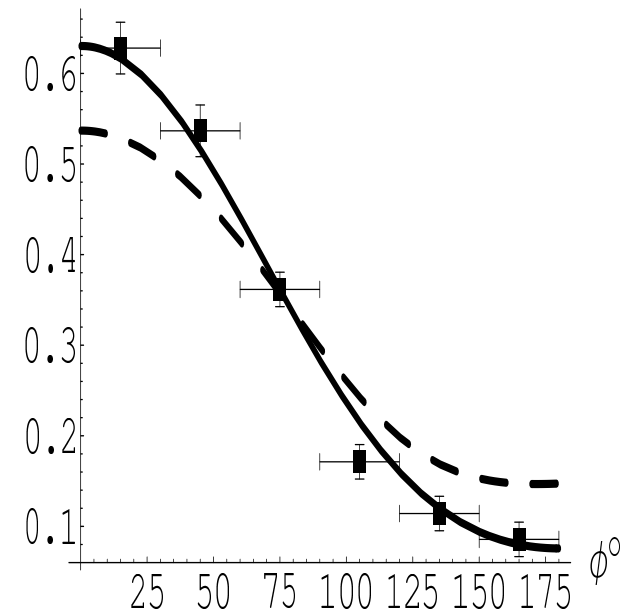

$d \sigma_{p p \rightarrow p X p} / d \phi / \sigma_{p p \rightarrow p x p}$

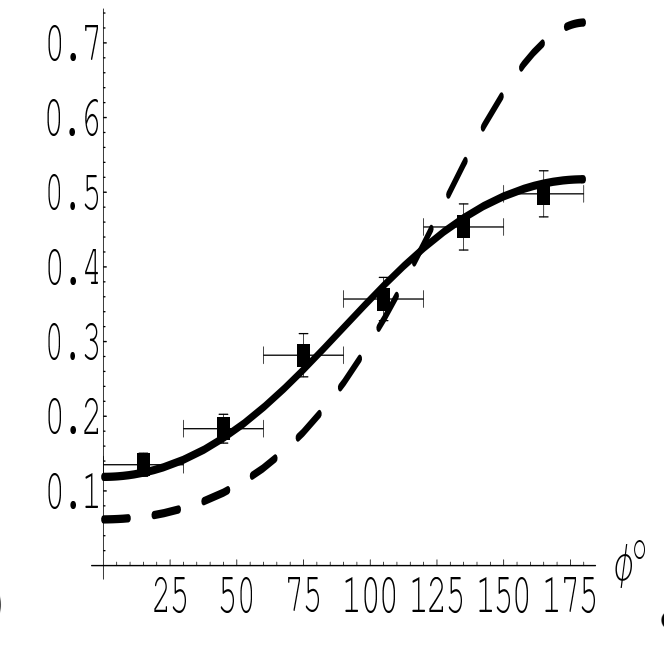

$d \sigma_{p p \rightarrow p x p} / d \phi / \sigma_{p p \rightarrow p X p}$

b)

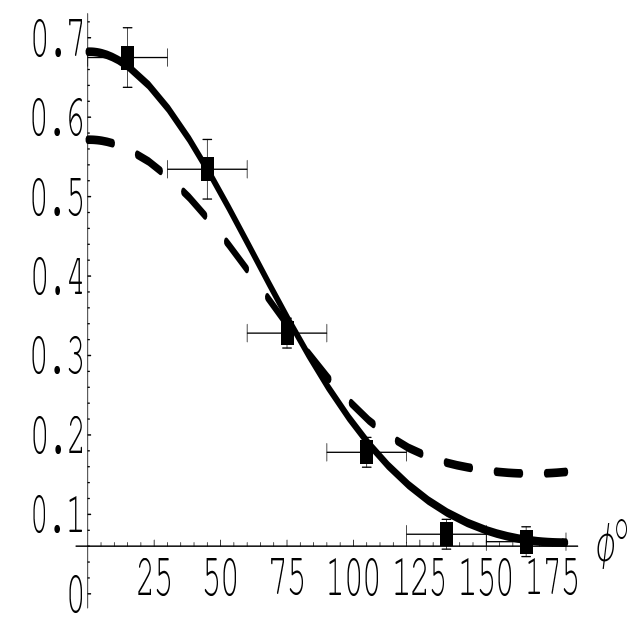

$d \sigma_{p p \rightarrow p X p} / d \phi / \sigma_{p p \rightarrow p x p}$

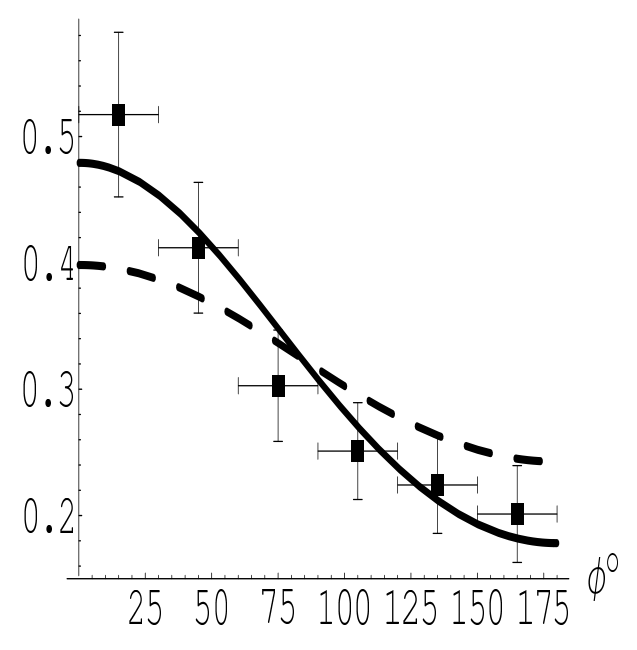

Figure 4: 
$d \sigma_{p p \rightarrow p X p} / d \phi / \sigma_{p p \rightarrow p X p}$

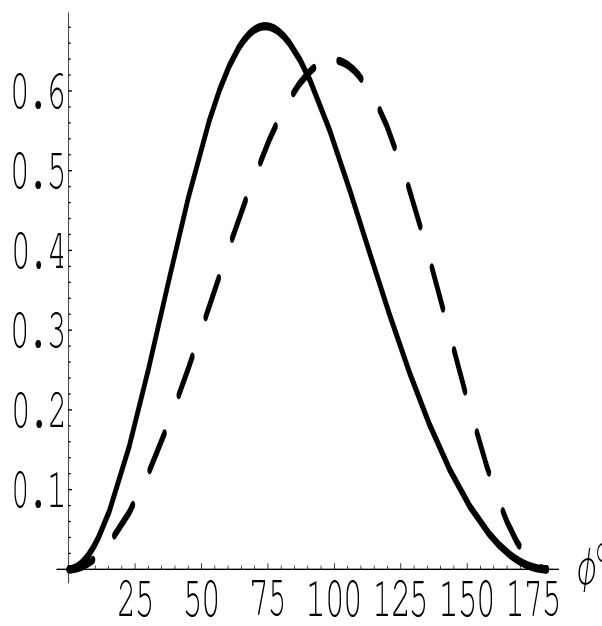

a)

$d \sigma_{p p \rightarrow p X p} / d \phi / \sigma_{p p \rightarrow p X p}$

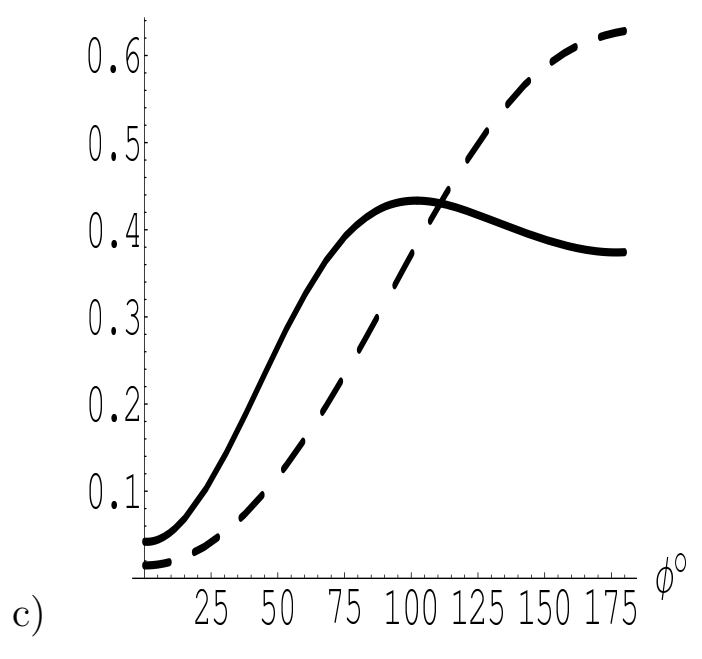

$d \sigma_{p p \rightarrow p X p} / d \phi / \sigma_{p p \rightarrow p x p}$

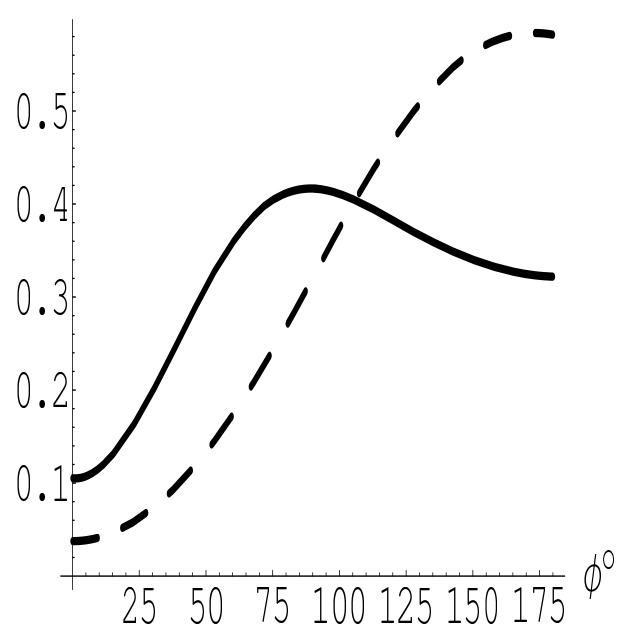

$d \sigma_{p p \rightarrow p x p} / d \phi / \sigma_{p p \rightarrow p x p}$

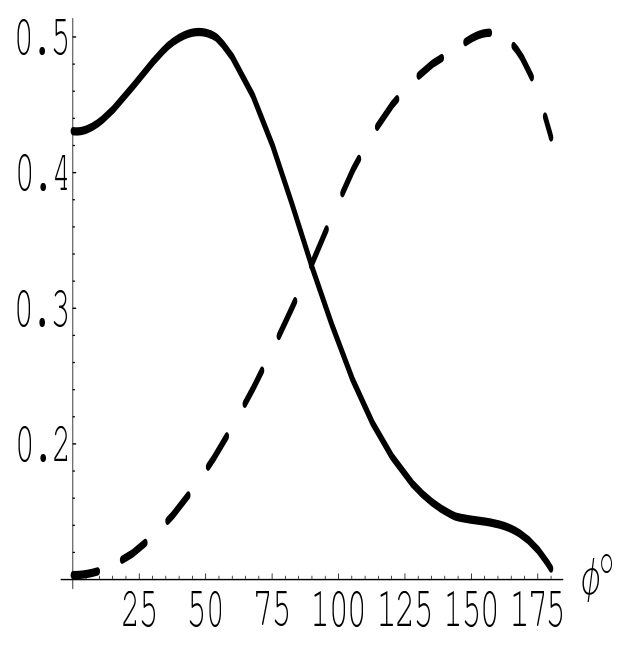

Figure 5: 


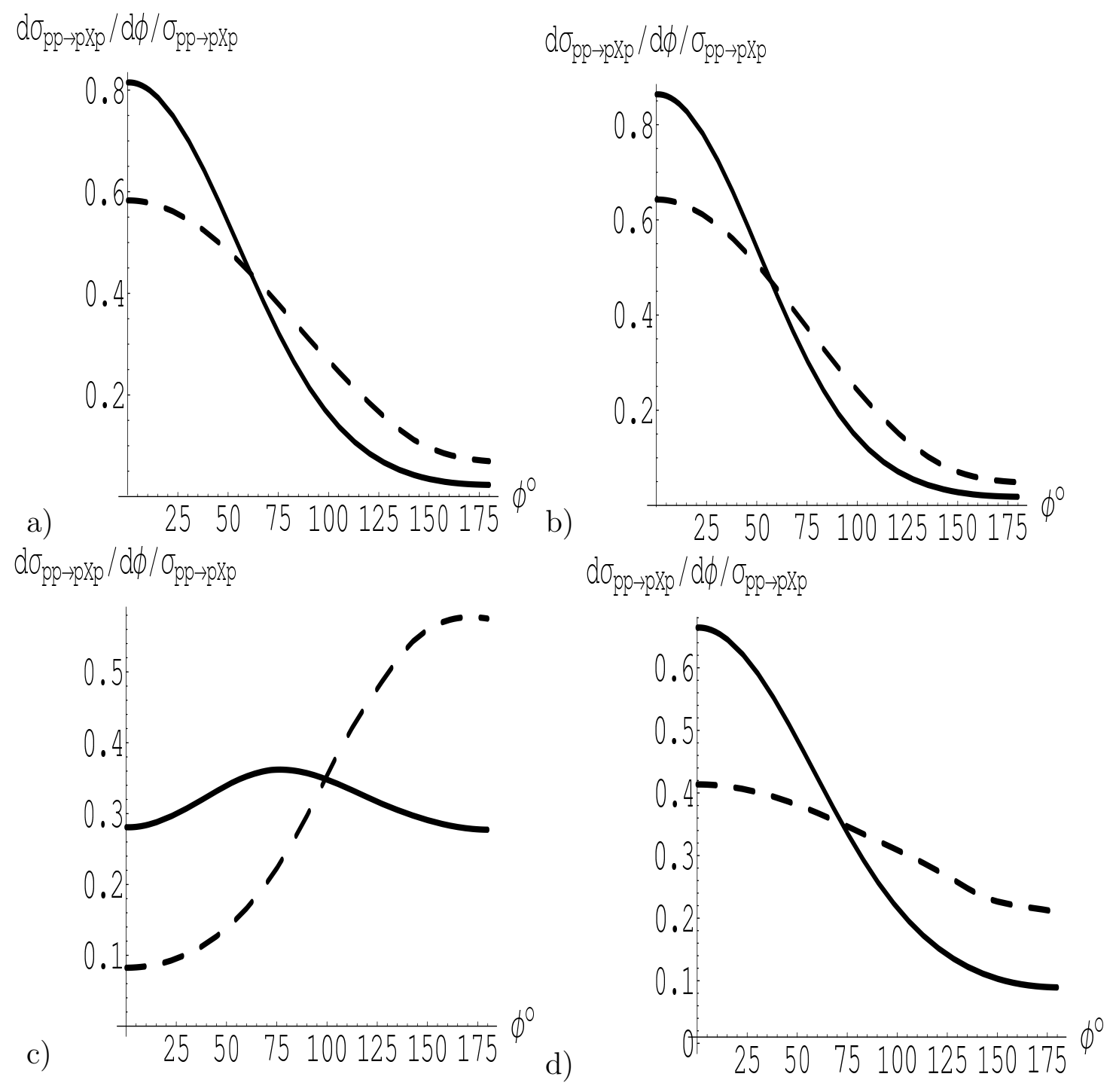

Figure 6: 

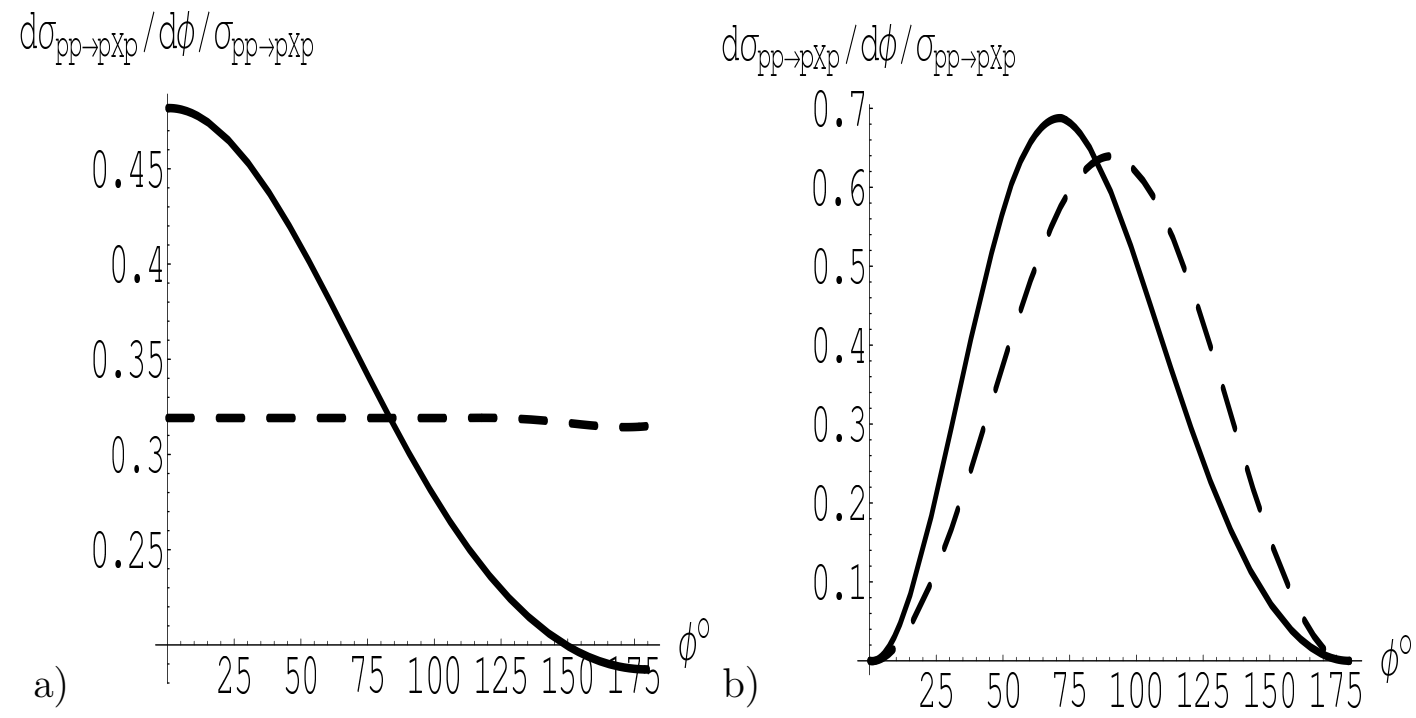

Figure 7: 